\title{
The Spanish and Portuguese Keyboards, the best options to type in all Romance Languages for US- QWERTY users
}

\author{
Enrique Tébar, $\mathrm{PhD}$ \\ Honorary Collaborator - Dpt. of Physics, Systems Engineering and Sign Theory \\ University of Alicante, Spain - Address: Carretera de San Vicente del Raspeig, $s / n$, \\ 03690 San Vicente del Raspeig, Alicante, Spain - e-mail: enrique.tebar@ua.es
}

\section{Table of contents}

Abstract 1

1.Introduction 2

2.Latin Alphabet, Keyboards and Special Characters. 3

3.QWERTY and AZERTY Keyboards 4

4.Compatibilities for every Language and Keyboard type 5

4.1.Spanish Keyboard for Special Characters needed in Romance Languages...................... 6

4.2.Portuguese Keyboard for Special Characters needed in Romance Languages.................. 8

4.3.Italian Keyboard for special characters needed in Romance Languages ......................... 9

4.4. Summary of compatibilities Keyboards/Languages ................................................ 10

4.5. Spanish, Portuguese and Italian Keyboards for Punctuation Marks in an US English Keyboard.....

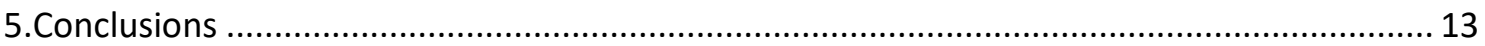

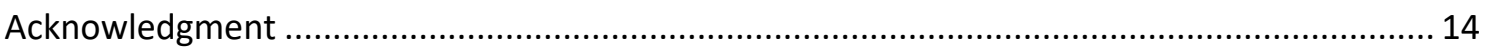

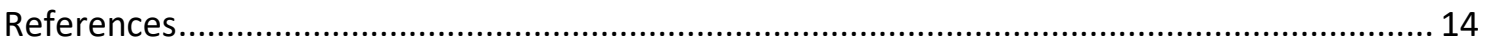

\section{Abstract}

While adequate for English-speaking users in the United States, as well as many Commonwealth countries and other English-speaking jurisdictions (e.g., Canada, Australia, New Zealand or South Africa among others), typing in Romance Languages (Spanish, French, Portuguese and Italian) by using a standard US-QWERTY Keyboard is not easy since it is not adapted to special characters such as accented vowels, tildes and cedillas or ligatures, used in Romance Languages. With regard to the International Layout, intended to enable access to the most common diacritics used in Western European Languages, the problem comes from the fact that accented vowels are spread throughout the Keyboard layout, and their uppercase versions need chord combinations which can require good manual dexterity.

This paper will analyze how the Spanish or Portuguese Keyboards are the best options for these users since they are they are QWERTY-based and the most compatible ones for the different character sets in Spanish, French, Portuguese and Italian Languages. 


\section{Introduction}

As the phenomenon of globalization becomes more and more present in each and every one of the social and commercial relations, the learning of foreign languages is also becoming more and more popular. According to [1], English is the first language in the world by attending to total number of speakers (both native and foreign Language speakers), followed closely by Mandarin Chinese, except that this one is just official in People's Republic of China, whilst English is, according to data updated in 2019, official Language in 55 sovereign states and 27 non-sovereign entities:

Table 1. Top 10 of Languages by total number of speakers

\begin{tabular}{|c|c|c|c|c|c|c|c|c|}
\hline \multirow{2}{*}{ Rank } & Language & Family & Branch & \multicolumn{2}{|c|}{ First Language (L1) } & \multicolumn{2}{c|}{ Second Language (L2) } & $\begin{array}{c}\text { Total No. } \\
\text { of speakers }\end{array}$ \\
\cline { 5 - 9 } & & & No. Of speakers & Rank & $\begin{array}{c}\text { No. Of speakers } \\
\text { (in millions) }\end{array}$ & Rillions) & Rank & (in millions) \\
\hline 1 & English & Indo-European & Germanic & 369.7 & 3 & 898.4 & 1 & 1268 \\
\hline 2 & $\begin{array}{c}\text { Mandarin Chinese } \\
\text { (incl. Standard } \\
\text { Chinese) }\end{array}$ & Sino-Tibetan & Sinitic & 921.5 & 1 & 198.7 & 4 & 1120 \\
\hline 3 & Hindi & Indo-European & Indo-Aryan & 342.0 & 4 & 295.3 & 2 & 637.3 \\
\hline 4 & Spanish & Indo-European & Romance & 463.0 & 2 & 74.9 & 9 & 537.9 \\
\hline 5 & French & Indo-European & Romance & 77.3 & 15 & 199.3 & 3 & 276.6 \\
\hline 6 & Standard Arabic & Afro-Asiatic & Semitic & N/A & N/A & N/A & N/A & 274.0 \\
\hline 7 & Bengali & Indo-European & Indo-Aryan & 228.5 & 5 & 36.8 & 13 & 265.2 \\
\hline 8 & Russian & Indo-European & Slavic & 153.6 & 7 & 104.3 & 6 & 258.0 \\
\hline 9 & Portuguese & Indo-European & Romance & 227.9 & 6 & 24.2 & 15 & 252.2 \\
\hline 10 & Indonesian & Austronesian & $\begin{array}{c}\text { Malayo- } \\
\text { Polynesian }\end{array}$ & 43.6 & 24 & 155.4 & 5 & 199.0 \\
\hline
\end{tabular}

It is also a fact that 3 Languages among these Top 10 share alphabet with English and are also spoken at neighbor countries from English-speaking ones. We are referring obviously to Spanish, French and Portuguese. Besides, some countries as Canada or Cameroon have both English and French as official languages and in some others as Puerto Rico both English and Spanish share officiality. And beyond the co-officiality, a lot of speakers from United States, Trinidad and Tobago, Belize or Guyana are able to speak and write in these 2 Languages ${ }^{2}$.

And it is also a fact that, as new technologies become more and more usual in our lives, the possibility of learning foreign Languages becomes easier, as well as the need of typing in several Languages with our computers, tablets and smartphones, given that the number of students in Spanish, French and Portuguese as foreign languages is also increasing according to studies made by Instituto Cervantes ${ }^{3}$, L'Observatoire de la Langue Française ${ }^{4}$ and Instituto Camões ${ }^{5}$ respectively.

Finally, Italian Language ranks position number $27^{\text {th }}$ in the classification made by [1], with 67.7 million speakers all over the world. This is the $4^{\text {th }}$ Romance Language according to this classification and jointly with Spanish, French and Portuguese will be also considered for this study, where the Spanish Keyboard will be determined as the best option to type in all Romance Languages for US-QWERTY users.

The manuscript comes up from the author's experience in International Project Managing of Solar Photovoltaics when working in foreign countries belonging to Commonwealth and Francophonie by using local systems and working together with local mates. The author worked with his own Spanish laptop and discovered that local 
users could easily get on with the author's Spanish Keyboard to type also in English and French Languages, even better than with their own laptops, supposedly adapted to their mother tongues.

\section{Latin Alphabet, Keyboards and Special Characters}

The Latin alphabet is shared by English, Spanish, French, Portuguese and Italian Languages, so the main characters are therefore shared by all of them, and every language counts with its own Keyboard, adapted to the writing needs for this language.

English Language has no special characters beyond common letters (from A to Z, capital and lowercase), numbers and punctuation and symbol keys found on a traditional typewriter. So, typing in English Language can be easily made by using either a Spanish, French, Portuguese or Italian Keyboard.

However, the 4 most spoken Romance Languages count with special characters needed to type correctly in the languages addressed in this paper, that cannot be directly obtained by using an US or UK English Keyboard. These special characters are the following:

- Spanish:

○ Letter "Eñe" (Ñ,$\tilde{n})$.

- Acute accent over the 5 vowels (Á, É, Í, Ó, Ú, capital and lowercase).

- Umlaut (also known as Diaeresis or Tréma) over the vowel U (Ü, ü).

- French:

- Letter "C Cédile" (Ç, ç).

- Letter "Ethel" or Ligature "oe" ( $($ E, œ).

- Acute accent over the vowel $E(E$, é).

- Grave accent over the vowels A, E, U (À, È, Ù, capital and lowercase).

- Circumflex accent over the 5 vowels (Â, Ê, Î, Ô, Û, capital and lowercase).

- Umlaut over the vowels E, I, U (Ë, Ï, Ü capital and lowercase).

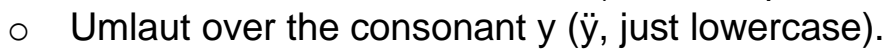

- Portuguese:

- Letter "C Cedilha" (Ç, ç).

- Acute accent over the 5 vowels (Á, É, Í, Ó, Ú, capital and lowercase).

- Grave accent over the vowel $A(\grave{A}$, à).

- Til accent over the vowels $A, O(\tilde{A}, \tilde{O}$, capital and lowercase).

- Circumflex accent over the vowels $A, E, O(\hat{A}, \hat{E}$, Ô, capital and lowercase).

- Italian:

- Acute accent over the vowel E (É, é).

- Grave accent over the 5 vowels (À, È, Ì, Ò, Ù, capital and lowercase).

- Circumflex accent over the vowel I (Î, î).

All these special characters belong to the extended ASCII table from ASCII Code ${ }^{6}$. This means that all of them can be obtained by typing simultaneously the letter "Alt" and the following combinations typed with the numeric keypad. So, e.g. "N"N" can be obtained by typing Alt + 0165, "ê" by typing Alt + 0136 and "œ" by typing Alt + 0156 . 
Unfortunately, they won't work on a laptop without numeric keypad, unless the function "Num Lock" is activated, which can cause a big hassle because then the letters will not work until the function "Num Lock" is switched off.

It may seem that, given that French Language is the Romance Language containing more special characters among the 4 most spoken ones, French Keyboard will be the most standard one to write as best as possible in all of them. However, nothing could be more untrue. Firstly, because French Keyboard is AZERTY-based, contrarily to the rest of Keyboards for the rest of Romance Languages, which are QWERTY-based, as will be described next. Secondly, because some characters needed to write correctly in French Language do not even appear in French Keyboard but in Spanish and Portuguese ones.

\section{QWERTY and AZERTY Keyboards}

The so-called "QWERTY" layout was designed in 1868 by Christopher Sholes (the inventor of the Typewriter) in the United States and remains in ubiquitous use up to present. The way how the letters are distributed along and across the layout is due to its designer's criteria. Thus, Sholes arranged the keys in their odd fashion to prevent jamming on mechanical typewriters by separating commonly used letter combinations. ${ }^{7}$

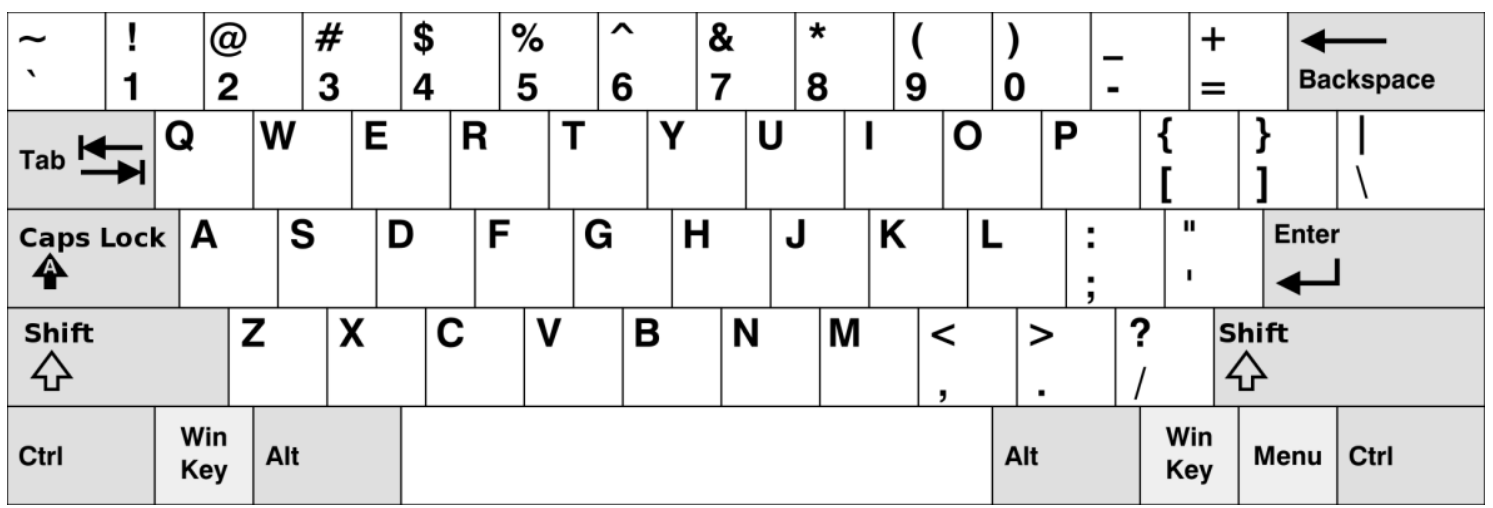

Fig. 1 - QWERTY (United States) Keyboard layout ${ }^{8}$

QWERTY layout was inherited by personal computers but given that QWERTY layout was designed for English Language, without accents, the standard US Keyboard has no provision for it at all; the need for other languages was later met by adding the socalled "dead keys" to type accents without having to add more keys. ${ }^{10}$

QWERTY standard was followed by the majority of Western countries with the exceptions of:

- German-speaking countries (mainly Germany, Austria and Switzerland), that switched the positions of $Y$ and $Z$ letters creating the so-called "QWERTZ" layout because " $Z$ " is a much more common letter than " $Y$ " in Germany and because "T" and "Z" often appear next to each other in the German orthography, and typewriter jamming would be reduced by placing the two keys so they could be typed with separate hands. ${ }^{11}$ Some other Non-Latin Central European countries such as Czech Republic, Slovakia, Hungary and Poland, have also followed QWERTZ layout. However, given that this paper is based on English and Romance Languages, German Keyboard will not be considered in our discussion. 
- France and Belgium, that created a different standard called "AZERTY" during the last decade of the 19th century as a variation of QWERTY typewriters. ${ }^{12}$ This variation pretends to make easier the typing in French Language by placing the numbers 0 to 9 in the "Caps" position of the top row to make easier the typing of accented vowels and lowercase "ç" and by switching letters " $A$ " and " $Q$ ", by switching letters " $W$ " and " $Z$ " and by placing " $M$ " letter right to " $L$ ", according to this layout:

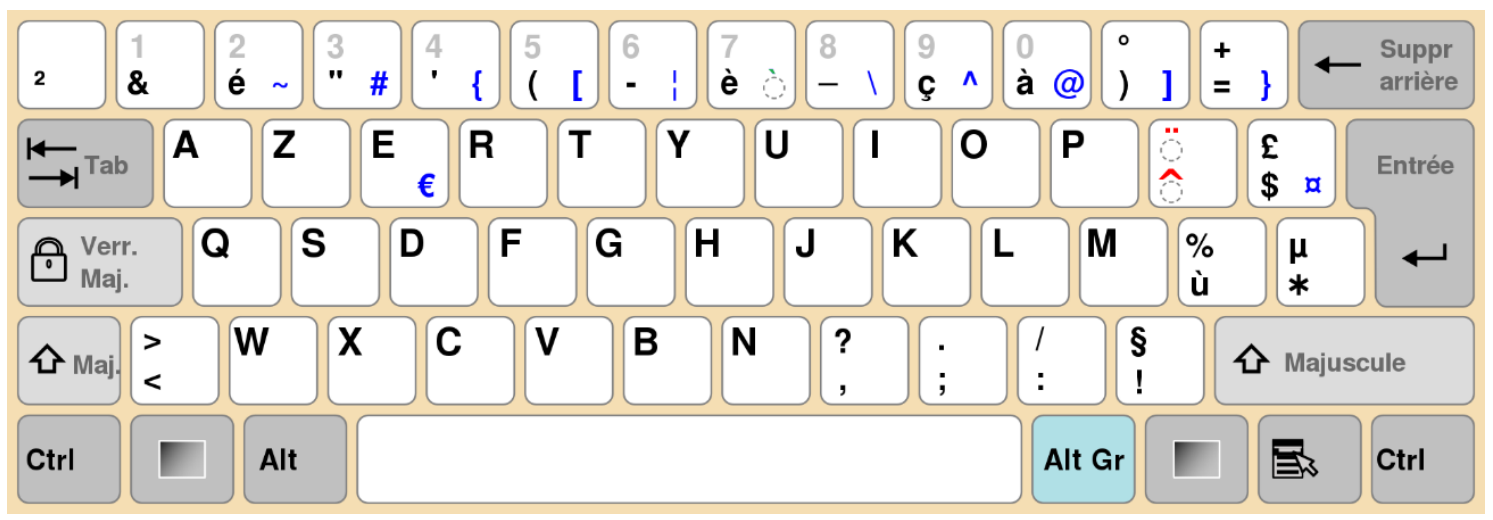

Fig. 2 - AZERTY (France) Keyboard layout ${ }^{13}$. Dead keys appear in red color and characters obtained by pressing "Alt Gr" simultaneously appear in blue and the sole case of both " " (dead key + "Alt Gr") appears in green.

It is not easy for a QWERTY user to switch to an AZERTY layout depending on the language to create a document, because the variations between both are quite important. In fact, 5 letters ("A", "M", "Q", "W" and "Z") are shifted from the positions where the QWERTY user tries instinctively to find them. Moreover, paradoxically, French Keyboard does not count with keys to type capital C Cédille ("Ç"), uppercase accented "É" and ligature "œ" in any version (neither lower nor uppercase), all these characters can only be obtained by typing extended ASCII codes.

So, definitely, French Keyboard is not a convenient option for a US-QWERTY user who wants to type in a Romance Language, may it be French, Spanish, Portuguese or Italian.

Next chapter will analyze how adapted among them are the other 3 Keyboard layouts (Spanish, Portuguese and Italian), as well as to French Language.

\section{Compatibilities for every Language and Keyboard type}

As discussed in chapter 2, typing in English Language can be made by using either a Spanish, French, Portuguese or Italian Keyboard with no need of typing extended ASCII codes. So, no discussion about adaptation with English Language will be done in this chapter, except for Punctuation Marks such as point, comma, colon, semicolon or brackets, among others, that will be done in subchapter 4.5.

The difficulties to get used to a French Keyboard have also been discussed in chapter 3.

Now it is the time to check how efficient Spanish, Portuguese and Italian Keyboards, who are QWERTY-based are to type documents both in English and in any Romance Language. 
Tables presented in next subchapters $4.1,4.2$ and 4.3 shows how compatible the Spanish, Portuguese and Italian Keyboards are to type special characters needed in Romance Languages, where:

- " " " sign means that the keyboard is fully compatible with this special character (direct or dead key present at the keyboard).

- " $x$ " sign means that the character is not fully compatible ("Alt Gr" key or Ascii code are needed to generate it).

- Grey box means that the character does not exist in this language.

Both symbols ("V" and "x") will also be used for table shown in subchapter 4.5.

Subchapter 4.4. summarizes the compatibilities between the different Romance Keyboards and Romance Languages, as well as English.

\subsection{Spanish Keyboard for Special Characters needed in Romance Languages}

Figure 3 shows the Keyboard layout used in Spain. It includes letter "Ñ" placed right to "L" and dead keys for the 3 kinds of accents used in France, as well as the Umlaut, and the " $C$ Cédille". The reason why these characters not present in Spanish Language (Grave and Circumflex accents, as well as "Ç") are present in the Spanish Keyboard is because this keyboard layout is used to write in Spanish and in other languages of Spain such as Catalan, Basque, Galician, Aragonese, Asturian and Occitan, some of which need these special characters.

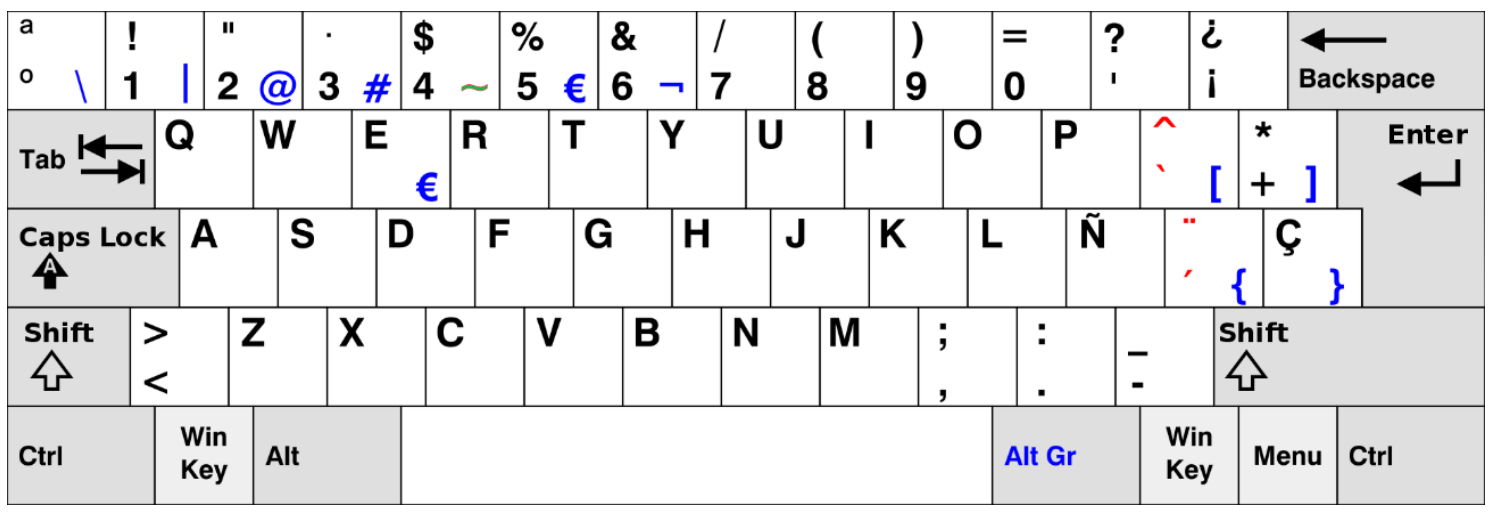

Fig. 3-QWERTY (Spain) Keyboard layout ${ }^{14}$. Dead keys appear in red color, characters obtained by pressing "Alt Gr" simultaneously appear in blue and the sole case of both " " (dead key + "Alt Gr") appears in green.

Oddly, Spanish Keyboard also includes the Til accent ( ) used in Portuguese over the vowels "A" and "O" and in Spanish to create the "N" from the "N". This Til accent acts as a dead key just over letters " $A$ ", "O" and "N" to create "Ã", "Õ" and "Ñ" characters respectively, whilst for the rest of letters this key combination generates 2 separate characters (e.g. " $\sim E$ ", " U"," $\sim P$ "). This Til accent ( ) is the unique "Alt Gr dead key" case and it can be obtained by pressing "Alt Gr" +4 . However, some Keyboard manufacturers don't mark this character at the "4" key, so this fact is unknown for the vast majority of Spanish QWERTY users.

The Spanish Latin American sort, used throughout Mexico, Central and South America is missing "Ç" key, as well as dead keys for Grave and Circumflex accents. This is why this sort is not especially compatible to type in French Language.

Table 2 shows that Spanish Keyboard is fully compatible with: 
Table 2. Compatibily of European Spanish or Brazilian Portuguese Keyboard for special characters needed in Romance Languages

\begin{tabular}{|c|c|c|c|c|}
\hline Special character & Spanish & French & Portuguese & Italian \\
\hline Á & $\sqrt{ }$ & & $\sqrt{ }$ & \\
\hline á & $\sqrt{ }$ & & $\sqrt{ }$ & \\
\hline É & $\sqrt{ }$ & $\sqrt{ }$ & $\sqrt{ }$ & $\sqrt{ }$ \\
\hline é & $\sqrt{ }$ & $\sqrt{ }$ & $\sqrt{ }$ & $\sqrt{ }$ \\
\hline Í & $\sqrt{ }$ & & $\sqrt{ }$ & \\
\hline í & $\sqrt{ }$ & & $\sqrt{ }$ & \\
\hline ó & $\sqrt{ }$ & & $\sqrt{ }$ & \\
\hline ó & $\sqrt{ }$ & & $\sqrt{ }$ & \\
\hline Ú & $\sqrt{ }$ & & $\sqrt{ }$ & \\
\hline ú & $\sqrt{ }$ & 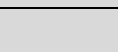 & $\sqrt{ }$ & \\
\hline$\grave{A}$ & & $\sqrt{ }$ & $\sqrt{ }$ & $\sqrt{ }$ \\
\hline à & 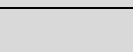 & $\sqrt{ }$ & $\sqrt{ }$ & $\sqrt{ }$ \\
\hline$\grave{\mathrm{E}}$ & & $\sqrt{ }$ & & $\sqrt{ }$ \\
\hline è & & $\sqrt{ }$ & 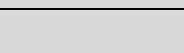 & $\sqrt{ }$ \\
\hline Ì & & & & $\sqrt{ }$ \\
\hline ì & & 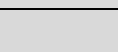 & 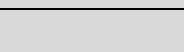 & $\sqrt{ }$ \\
\hline Ò & & 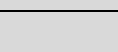 & 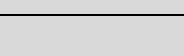 & $\sqrt{ }$ \\
\hline ò & & 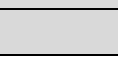 & 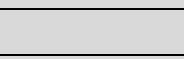 & $\sqrt{ }$ \\
\hline Ù & & $\sqrt{ }$ & 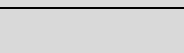 & $\sqrt{ }$ \\
\hline ù & & $\sqrt{ }$ & 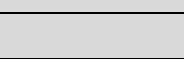 & $\sqrt{ }$ \\
\hline$\hat{A}$ & & $\sqrt{ }$ & $\sqrt{ }$ & \\
\hline$\hat{a}$ & & $\sqrt{ }$ & $\sqrt{ }$ & \\
\hline$\hat{\mathrm{E}}$ & & $\sqrt{ }$ & $\sqrt{ }$ & \\
\hline$\hat{\mathrm{e}}$ & & $\sqrt{ }$ & $\sqrt{ }$ & 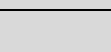 \\
\hline$\hat{\imath}$ & & $\sqrt{ }$ & 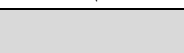 & $\sqrt{ }$ \\
\hline$\hat{\imath}$ & & $\sqrt{ }$ & 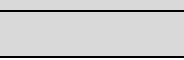 & $\sqrt{ }$ \\
\hline Ô & & $\sqrt{ }$ & $\sqrt{ }$ & \\
\hline$\hat{o}$ & & $\sqrt{ }$ & $\sqrt{ }$ & \\
\hline$\hat{U}$ & & $\sqrt{ }$ & 1 & \\
\hline$\hat{\mathrm{u}}$ & & $\sqrt{ }$ & 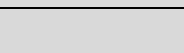 & \\
\hline$\ddot{\mathrm{E}}$ & & $\sqrt{ }$ & & \\
\hline ë & & $\sqrt{ }$ & 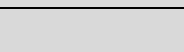 & \\
\hline$\ddot{i}$ & & $\sqrt{ }$ & 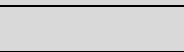 & \\
\hline$\ddot{i}$ & & $\sqrt{ }$ & & \\
\hline Ü & $\sqrt{ }$ & $\sqrt{ }$ & 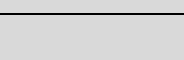 & \\
\hline ü & $\sqrt{ }$ & $\sqrt{ }$ & & \\
\hline$\ddot{y}$ & & $\sqrt{ }$ & 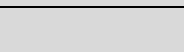 & \\
\hline$\tilde{A}$ & & & $\sqrt{ }$ & \\
\hline ã & & & $\sqrt{ }$ & \\
\hline$\tilde{O}$ & & & $\sqrt{ }$ & \\
\hline$\tilde{o}$ & & & $\sqrt{ }$ & \\
\hline$\tilde{\mathrm{N}}$ & $\sqrt{ }$ & & & \\
\hline$\tilde{n}$ & $\sqrt{ }$ & & & \\
\hline Ç & & $\sqrt{ }$ & $\sqrt{ }$ & \\
\hline ç & & $\sqrt{ }$ & $\sqrt{ }$ & \\
\hline $\mathcal{C F}$ & & $x$ & & \\
\hline$œ$ & & $x$ & & \\
\hline
\end{tabular}


- All the special characters needed in Spanish, Portuguese and Italian.

- $\quad$ All the special characters needed in French except the letter "Ethel" or Ligature "oe" ( $O E, œ)$, that is not compatible with French Keyboard either and can be obtained with Alt + 0140 ("OE") and Alt + 0156 ("œ"). It is also important to remark that Spanish Keyboard is fully compatible with all the accented vowels in French, both caps and lowercase, whilst standard versions of French Keyboard were unable to generate uppercase versions of accented vowels until the publication of the voluntary standard or norme volontaire NF Z71-300 made by AFNOR in $2019 .{ }^{15}$

\subsection{Portuguese Keyboard for Special Characters needed in Romance Languages}

Figures 4 and 5 show the Keyboard layout used in Brazil and Portugal respectively. Both are quite similar since all the Latin alphabet, including the "C Cedilha" is placing in the same position. The main difference is that the Brazilian version includes the Umlaut or Tréma as caps version of "6" key whilst the European Portuguese version leaves it at "Alt Gr" version of "*/+" key.

The reason why is because the Umlaut was used in Brazilian Portuguese to indicate that the letter " $U$ " in certain combinations where is normally mute, should be pronounced, but it is no longer written since the 2009 Spelling Reform, except in foreign names. ${ }^{16}$

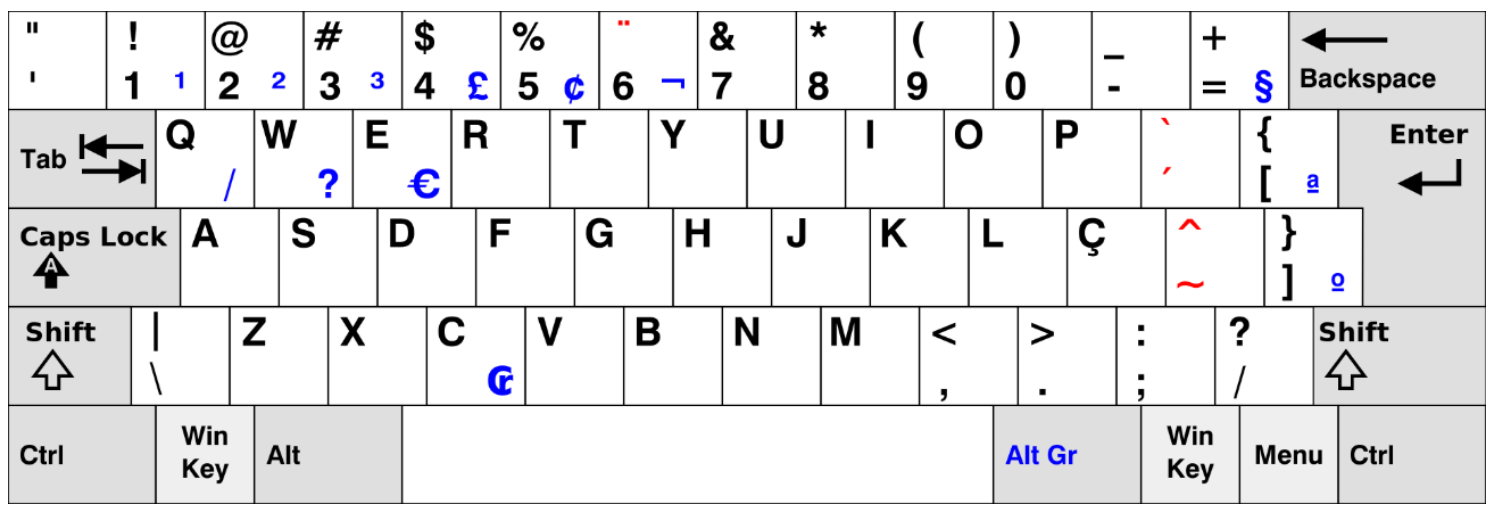

Fig. 4- QWERTY (Brazil) Keyboard layout ${ }^{17}$. Dead keys appear in red color and characters obtained by pressing "Alt Gr" simultaneously appear in blue.

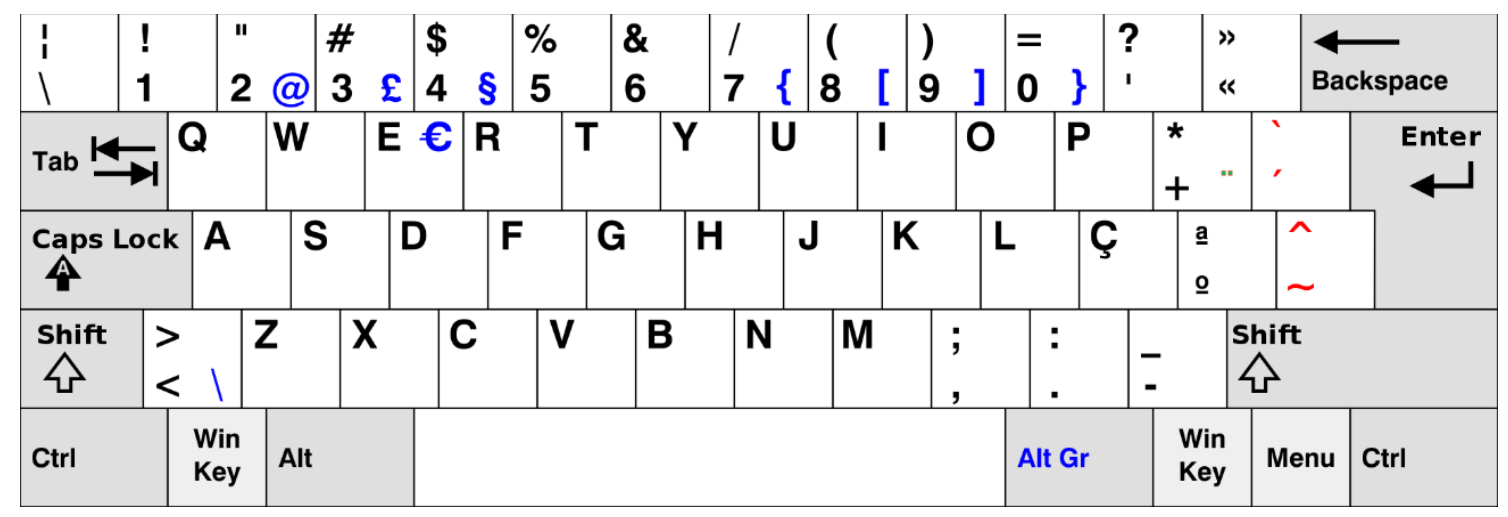

Fig. 5- QWERTY (Portugal) Keyboard layout ${ }^{18}$. Dead keys appear in red color, characters obtained by pressing "Alt Gr" simultaneously appear in blue and the sole case of both "'"” (dead key + "Alt Gr") appears in green. 
So, given that among Lusophone speakers, Brazilian represent more than $80 \%{ }^{19}$ and given that Brazilian Keyboard includes Umlaut as dead letter for Caps "6", we will consider Brazilian Keyboard to check the compatibility with special characters used in the 4 Romance Languages studied in this paper, the same way as it was done in previous subchapter with European Spanish Keyboard.

And we can conclude that the compatibility is the same as the European Spanish Keyboard, given that:

- Accented vowels with Acute, Grave, Circumflex, Umlaut and Til are easily available. So, all the accented vowels used in French, Spanish, Portuguese and Italian can be easily introduce by dead keys. Spanish Letter "N" is also fully compatible with Portuguese Keyboard by using dead key for Til accent and then "N" letter.

- French letter "Ç" is also fully compatible with Portuguese Keyboard.

- As explained in subchapter 4.1, the French letter "Ethel" that is not compatible with French Keyboard either, can be obtained in a Portuguese Keyboard with Alt +0140 or 0156 . Same as happens with the Spanish one, Portuguese Keyboard is fully compatible with all the accented vowels in French, both caps and lowercase, whilst standard versions of French Keyboard are not.

For the European Portuguese Keyboard, the conclusions are very similar with the sole exception of the Umlaut that in this version needs an "Alt Gr dead key", but the Umlaut appears very rarely in French and Spanish, so we can consider compatibilities of both Portuguese Keyboards practically the same.

\subsection{Italian Keyboard for special characters needed in Romance Languages}

This study ends with the Italian Keyboard layout, given that Italian is the $4^{\text {th }}$ Romance Language with respect to its number of speakers and Italian Keyboard, shown in Figure 6 , is also based on QWERTY standard.

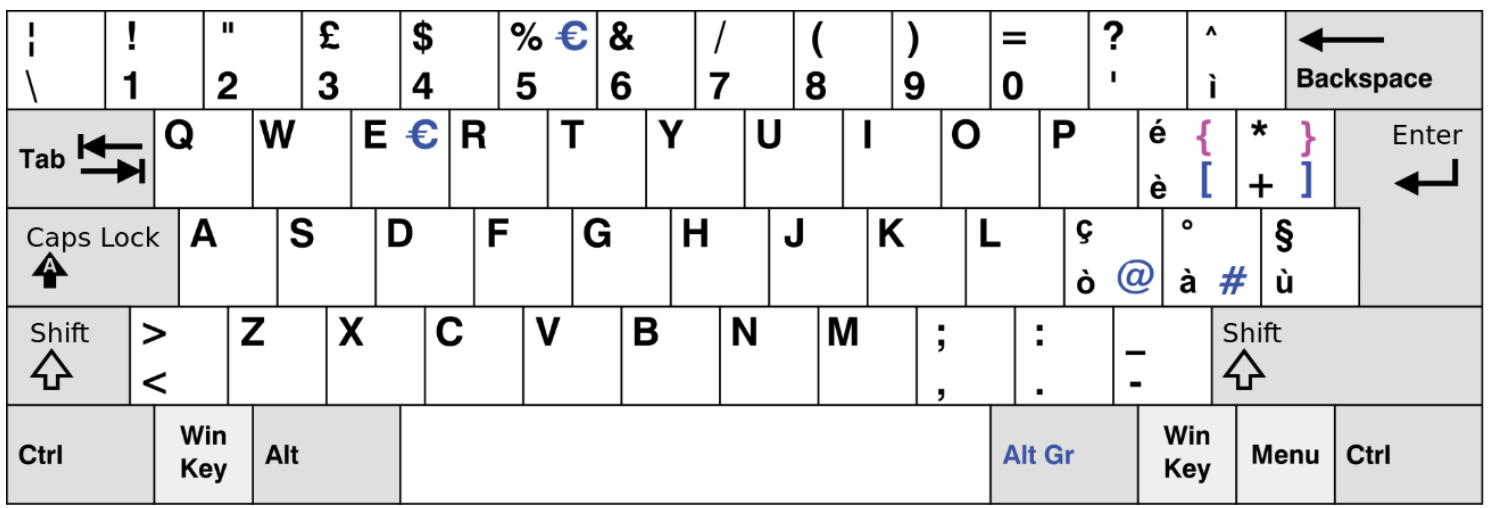

Fig. 6-QWERTY (Italy) Keyboard layout ${ }^{20}$. There are no dead keys. Characters obtained by pressing "Alt Gr" simultaneously appear in blue and Characters obtained by pressing "Shift" and "Alt Gr" simultaneously appear in purple.

At a glance we can see that the absence of dead keys just enables Italian Keyboard to generate the accented vowels that exist in Italian (Acute accent over the vowel é in lowercase, Grave accent over the 5 vowels, just lowercase), but no Circumflexed "î". In fact, the Circumflex symbol placed in Caps position of "i" letter is not a dead key but a generator of the character " $\wedge$ " with no vowel above. So, Italian Keyboard, same as it happened with French one, is not $100 \%$ compatible with the Language it is supposed 
to be designed for. Oddly, Italian Keyboard counts with "ç" character (lowercase version) that exists in French and Portuguese but not in Italian.

Given that the Italian Keyboard is not fully compatible with Italian Language itself, a compatibility table of Italian Keyboard with the rest of Romance Languages (Spanish, French and Portuguese) will not be made either. However, a general table of compatibilities between all keyboards and languages studied will be shown next.

\subsection{Summary of compatibilities Keyboards/Languages}

From the previous subchapters we can summarize the table 3, shown at next page.

Color code to show the compatibility degree is the following:

- Green: Full or Very High Compatibility.

- Blue: High Compatibility.

- Orange: Low Compatibility.

- Red: Very Low Compatibility.

We can check that the recommended Keyboards to write in the following Languages are:

- To write in English: All of them are valid.

- To write in Spanish: Just Spanish or Portuguese Keyboards are fully compatible.

- To write in French: The best options are Spanish or Portuguese Keyboards, followed by French.

- To write in Portuguese: Just Portuguese or Spanish Keyboards are fully compatible.

- To write in Italian: The best options are Spanish or Portuguese Keyboards, followed by French, and followed by Italian.

For bilingual needs or purposes (English + One Romance Language), given that all the Keyboards are valid to write in English, the user must check the compatibility with the applicable Romance Language. So, e.g. a US-QWERTY user who wants to type both in English and French, should choose a Spanish or Portuguese Keyboard as first option.

For multilingual needs or purposes (English + Two Romance Languages), the user must check the compatibility with the most restrictive Romance Language. So, e.g. a US-QWERTY user who wants to type in English, Spanish and French, should also choose a Spanish or Portuguese Keyboard as first option.

\subsection{Spanish, Portuguese and Italian Keyboards for Punctuation Marks in an US English Keyboard}

Finally, we must also verify how different the position of usual Punctuation Marks according to ${ }^{21}$ are if we compare US English and the different Romance Keyboards that have been analyzed. For Spanish, both versions (European Spanish and Latin American) will be considered. For Portuguese, also both versions (Brazilian and European Portuguese) will be considered. 


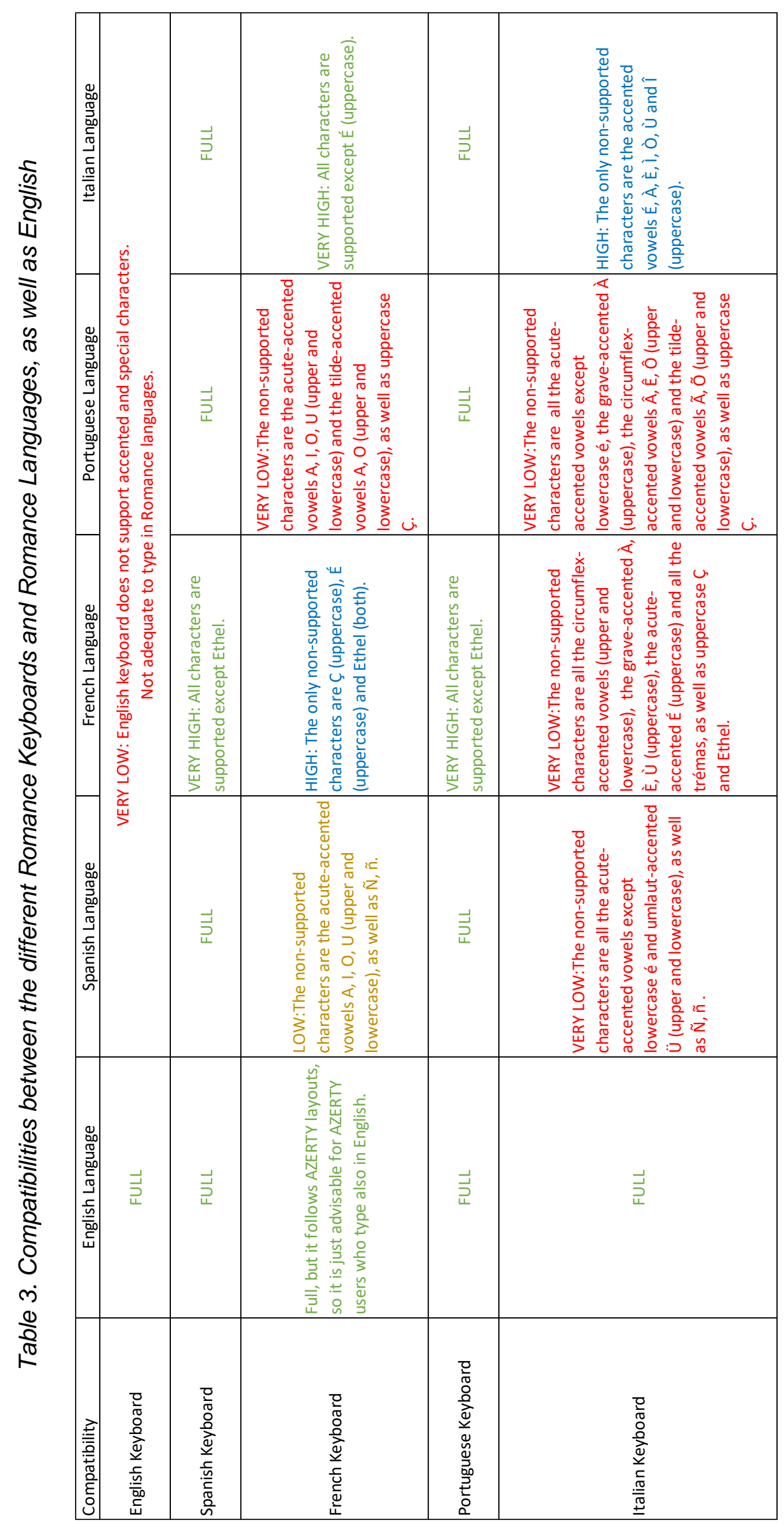


Table 4. Compatibility of the different Romance Keyboards with respect to the position of usual Punctuation Marks in US English layout

\begin{tabular}{|c|c|c|c|c|c|c|}
\hline \multirow{2}{*}{$\begin{array}{l}\text { Quotation mark } \\
\text { used in English }\end{array}$} & \multicolumn{6}{|c|}{ Position of the quotation mark in } \\
\hline & $\begin{array}{l}\text { Spanish } \\
\text { Keyboard }\end{array}$ & $\begin{array}{l}\text { Sp. LatAm } \\
\text { Keyboard }\end{array}$ & $\begin{array}{l}\text { French } \\
\text { Keyboard }\end{array}$ & $\begin{array}{c}\text { Por. Brazilian } \\
\text { Keyboard }\end{array}$ & $\begin{array}{c}\text { Portuguese } \\
\text { Keyboard }\end{array}$ & $\begin{array}{l}\text { Italian } \\
\text { Keyboard }\end{array}$ \\
\hline Period. & $\sqrt{ }$ & $\sqrt{ }$ & $x$ & $\sqrt{ }$ & $\sqrt{ }$ & $\sqrt{ }$ \\
\hline Question mark? & $x$ & $x$ & $x$ & $x$ & $x$ & $x$ \\
\hline Exclamation point! & $\sqrt{ }$ & $\sqrt{ }$ & $x$ & $\sqrt{ }$ & $\sqrt{ }$ & $\mathrm{V}$ \\
\hline Comma , & $\sqrt{ }$ & $\sqrt{ }$ & $x$ & $\sqrt{ }$ & $\sqrt{ }$ & $\mathrm{V}$ \\
\hline Semicolon ; & $x$ & $x$ & $x$ & $x$ & $x$ & $x$ \\
\hline Colon : & $x$ & $\times$ & $\times$ & $x$ & $x$ & $x$ \\
\hline Hyphen - & $x$ & $x$ & $x x$ & $\sqrt{ }$ & $\sqrt{ }$ & $x$ \\
\hline Underscore_. & $x$ & $x$ & $x x$ & $\sqrt{ }$ & $v$ & $x$ \\
\hline Apostrophe' & $x$ & $x$ & $x x$ & $x$ & $x$ & $x$ \\
\hline Quotation mark " & $x$ & $x$ & $x x$ & $x$ & $x$ & $x$ \\
\hline Dollar symbol \$ & V & $v$ & $x$ & $\sqrt{ }$ & $v$ & V \\
\hline Percent sign \% & $\sqrt{ }$ & V & $x$ & $\sqrt{ }$ & $v$ & V \\
\hline Ampersand \& & $x$ & $x$ & $x x$ & $\sqrt{ }$ & $x$ & $x$ \\
\hline At sign @ & $x x$ & $x x$ & $x x$ & $\sqrt{ }$ & $x x$ & $x x$ \\
\hline Number sign \# & $x x$ & 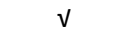 & $x x$ & $\sqrt{ }$ & V & $x x$ \\
\hline Plus sign + & $x$ & $x$ & $\sqrt{ }$ & $\sqrt{ }$ & $x$ & $x$ \\
\hline Equals sign = & $x$ & $x$ & $\sqrt{ }$ & $\sqrt{ }$ & $x$ & $x$ \\
\hline Asterisk * & $x$ & $x$ & $x$ & $\sqrt{ }$ & $x$ & $x$ \\
\hline Slash / & $x$ & $x$ & $x$ & $x$ & $x$ & $x$ \\
\hline Backslash \ & $x x$ & $x x$ & $x x$ & $x$ & $x$ & $x$ \\
\hline Vertical bar | & $x x$ & $x$ & $x x$ & $x$ & $x$ & $x$ \\
\hline Opening bracket ( & $x$ & $x$ & $x x$ & $\sqrt{ }$ & $x$ & $x$ \\
\hline Closing bracket ) & $x$ & $x$ & $x x$ & $\sqrt{ }$ & $x$ & $x$ \\
\hline Opening square bracket [ & $x x$ & $x$ & $x x$ & $x$ & $x x$ & $x$ \\
\hline Closing square bracket ] & $x x$ & $x$ & $x x$ & $x$ & $x x$ & $x$ \\
\hline Opening curly bracket \{ & $x x$ & $x$ & $x x$ & $x$ & $x \times$ & $x$ \\
\hline Closing curly bracket $\}$ & $x x$ & $x$ & $x x$ & $x$ & $x x$ & $x$ \\
\hline Opening angle bracket $<$ & $x$ & $x$ & $x$ & $\sqrt{ }$ & $x$ & $x$ \\
\hline Closing angle bracket > & $x$ & $x$ & $x$ & $\sqrt{ }$ & $x$ & $x$ \\
\hline Non-dead Tilde & $x x$ & $x x$ & $x x$ & $x x$ & $x x$ & $x x$ \\
\hline Non-dead Grave ` & $x x$ & $x x$ & $x x$ & $x x$ & $x x$ & $x x$ \\
\hline Non-dead Circumflex ${ }^{\wedge}$ & $x x$ & $x x$ & $x x$ & $x x$ & $x x$ & $x$ \\
\hline Total $\mathbf{V}$ & 5 & 6 & 2 & 17 & 8 & 5 \\
\hline Total $x$ & 16 & 21 & 12 & 12 & 16 & 23 \\
\hline Total $x \times$ & 11 & 5 & 18 & 3 & 8 & 4 \\
\hline
\end{tabular}

- " $\sqrt{ }$ " sign means that the position of usual punctuation mark is exactly the same as in US English, so a US-QWERTY user will get adapted with no effort.

- " $x$ " sign means that the position of usual punctuation mark is different to the one in US English, but the position is easy to find so a US-QWERTY user will get adapted with not too much effort. 
- " $x x$ " sign means that the position of usual punctuation mark is very different to the one in US English and the way to type this mark is hard to find out (usually "Alt Gr" key or a different criteria to place a number is needed), so a USQWERTY will need more effort to get adapted to this typing procedure.

\section{Conclusions}

From table shown in subchapter 4.5, we can easily deduct that, by far, Brazilian Portuguese Keyboard is the most friendly one for a US-QWERTY user to type both in English and in any Romance Language (Spanish, French, Portuguese or Italian), since among the 32 Punctuation Marks that are present in the US Keyboard:

- 17 are placed in the same position.

- $\quad 12$ are placed in a different position, but the introduction method is quite similar (easy adaptation).

- Just 3 are placed in a different position with a different introduction method, and they correspond to rarely used characters.

However, it is also a fact that the most used Punctuation Marks by far (period and comma), as well as the Exclamation Point, are placed in the same position in all the Romance Keyboards except French.

Besides, from table shown in subchapter 4.4, we can also deduct that European Spanish and European Portuguese Keyboards are fully compatible with Spanish, Portuguese, Italian and French Languages. So, all of them are adequate in general for bilingual and multilingual purposes for a US-QWERTY user who wants to type in a Romance Language (Typical profiles: Canadian user, bilingual in English and French, a US-QWERTY user who is learning a Romance Language or international trade relations).

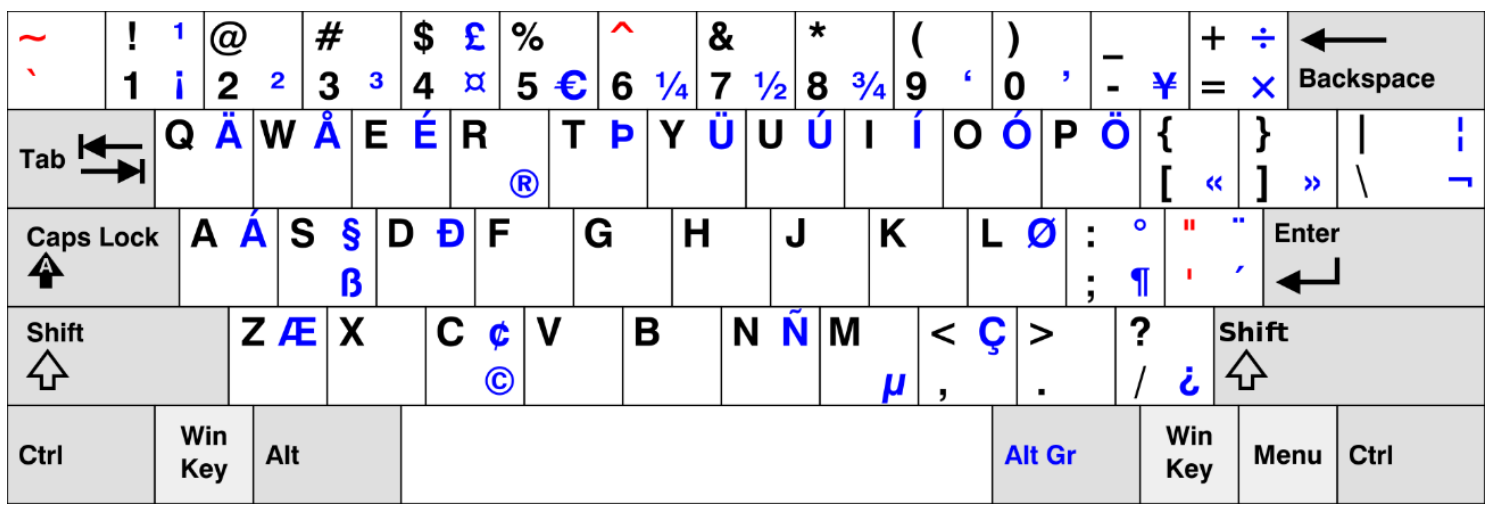

Fig. 7-QWERTY (United States) International Keyboard layout ${ }^{22}$

Switching to a Spanish or Portuguese Keyboard is very easy for a US QWERTY user, even more in case of Brazilian Portuguese Keyboard, as discussed. Acquiring a foreign keyboard at present is also relatively easy thanks to the numerous e-commerce companies that are present worldwide. And, in the author's opinion, getting used to a Spanish or Portuguese Keyboard is much easier in terms of Human-Computer Interaction than other techniques such as Virtual Keyboards or the so-called "USInternational Keyboard Layout", shown in Figure 7, intended to enable access to the most common diacritics used in Western European Languages, but not all of them. The problem comes from the fact that accented vowels are spread throughout the layout, 
and their uppercase versions need combinations Shift + Alt $\mathrm{Gr}+$ the letter to be accented, which can mean some chords that require additional manual dexterity.

\section{Acknowledgment}

The author wants to acknowledge the effort made by the programmers of Gate2home.com Virtual Online Keyboard, a very powerful tool that allows the user to easily verify how friendly the foreign Keyboard layout can be in terms of Human Interface Guidelines and Human-Computer Interaction.

\section{References}

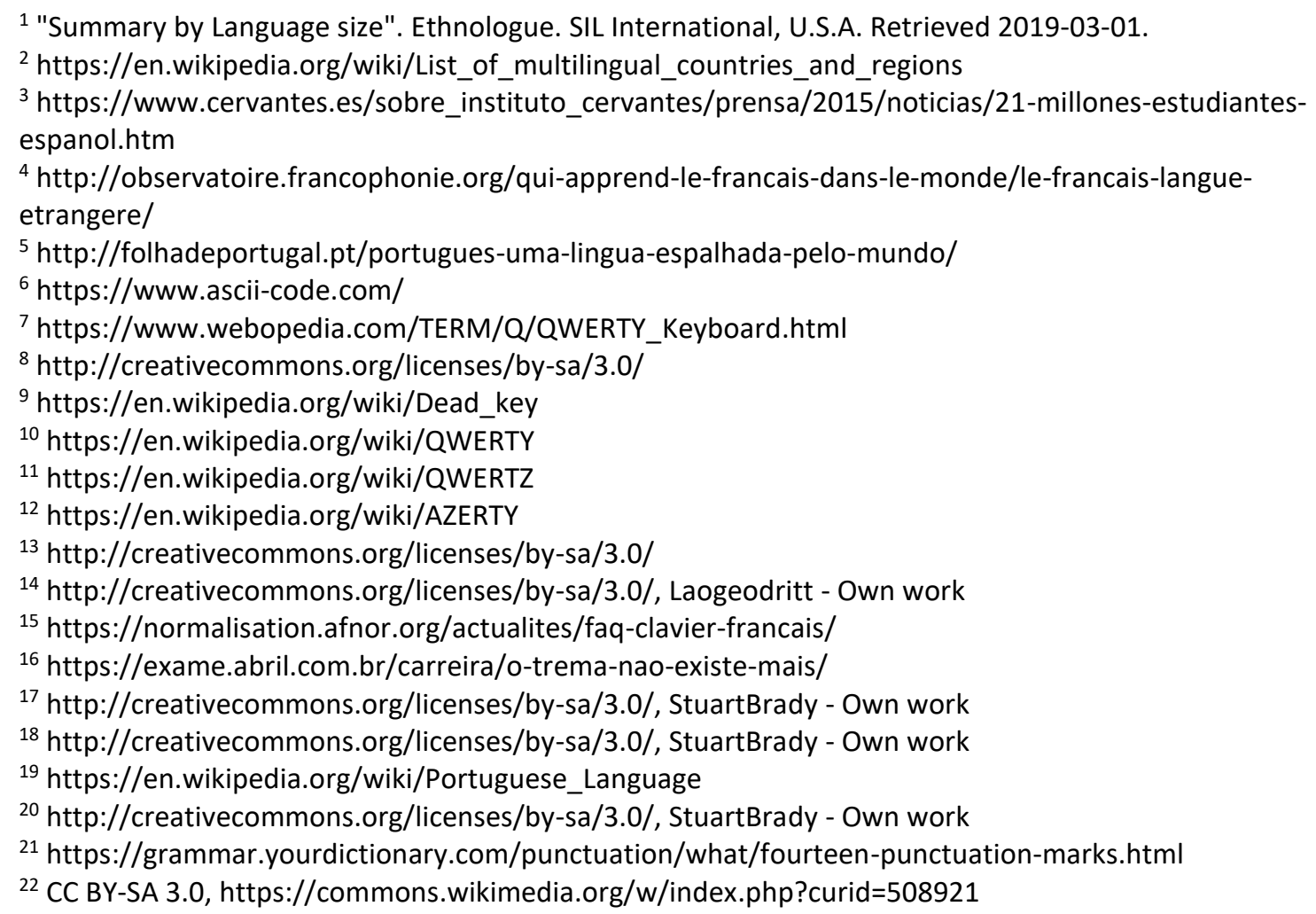

